\title{
The Use of Sucking Time to Establish Models to Estimate Breastmilk Intake of One Breastfeeding Session for 1-12-Month Infants: A Cross-sectional Study
}

Ni Jia

Capital Institute of Pediatrics

Mengxue Xu

Capital Institute of Pediatrics

Meixian Zhang

Enze Medical Research Center, Affiliated Taizhou Hospital of Wenzhou Medical College Jianhua Gong

Luohu Maternal and Child Health Hospital

\section{Lu Zhao}

Changping Maternal and Child Health Hospital

Yaohua Dai ( $\square$ yhdai2019@163.com)

Capital Institute of Pediatrics

\section{Research Article}

Keywords: Breastmilk intake, sucking time, estimation, infant, one breastfeeding session

Posted Date: September 20th, 2021

DOI: https://doi.org/10.21203/rs.3.rs-892243/v1

License: (c) (i) This work is licensed under a Creative Commons Attribution 4.0 International License.

Read Full License 
5 Ni Jia, Capital Institute of Pediatrics, No 2 Road Yabao, District Chaoyang, Beijing, China

6 Mengxue $\mathrm{Xu}$, Capital Institute of Pediatrics, No 2 Road Yabao, District Chaoyang, Beijing,

7 China

8 Meixian Zhang, Enze Medical Research Center, Affiliated Taizhou Hospital of Wenzhou

9 Medical College, No 150 Street Ximen, Linhai, Taizhou, Zhejiang, China

The Use of Sucking Time to Establish Models to Estimate Breastmilk Intake of One Breastfeeding Session for 1-12-Month Infants: A Cross-sectional Study Ni Jia, Mengxue Xu, Meixian Zhang, Jianhua Gong, Lu Zhao, Yaohua Dai Jianhua Gong, Luohu Maternal and Child Health Hospital, No 2013 Road Taibai, District Luohu, Shenzhen, China

Lu Zhao, Changping Maternal and Child Health Hospital, No 1 Road Beihuan, District Changping, Beijing, China

Yaohua Dai, Capital Institute of Pediatrics, No 2 Road Yabao, District Chaoyang, Beijing, China

Corresponding author: Yaohua Dai; address: No. 2 Road Yabao, District Chaoyang, Beijing, China; telephone number: +86 10 85695552; email address: yhdai2019@163.com. 


\section{Abstract}

Background: Observation method of assessing breastmilk intake (BI) by observing medical indices has less interference in breastfeeding than test-weighing method, but for the calculation certain influential predictor variables had not been recognized and needed to be further studied.

Method: Infants between 1 and 12 months were enrolled in a hospital of Shenzhen city in northern China. A cross-sectional survey was conducted in a sample of mother-infant dyads. A breastfeeding session of each participating mother subject was observed in the clinic. 150 mother-infant dyads were adopted for data analysis. BI was measured by test-weighing method. Sucking time (ST), Information of maternal and infant health, maternal breast, and infant feeding practice were collected.

Results: There was a relationship between sucking time and breastmilk intake per breastfeeding session $(r=0.57, \mathrm{p}<0.05)$. In the 4 multilevel models $(r>0.6, p<0.05)$ established to estimate breastmilk intake the best fit multilevel model was to estimate breastmilk intake per $\mathrm{kg}$ (infant weight) for $1-4$-month infants $\left(\mathrm{R}^{2}=0.53, \mathrm{p}<0.05\right)$. Sucking time as the key variable and other factors including infant birth weight/ birth weight, breast side, maternal BMI, maternal vocation significantly had effect on breastmilk intake and those factors that remained significant varied according to different age groups.

\section{Conclusions: Sucking time had a significant association with breastmilk intake in a} breastfeeding session. Establishing multilevel models based on sucking time to estimate breastmilk intake per infant weight for specific age group of infants greatly improved the effectiveness. But the accuracy of the estimation needed to be improved for further 
application in telemedicine.

\section{Keywords}

Breastmilk intake; sucking time; estimation; infant; one breastfeeding session

\section{Introduction}

Breastmilk is the optimal source for infants during the first 6 months of life, and breastfeeding has short-term and long-term beneficial effects on infant health and development. WHO Global Strategy recommended that infants should be exclusively breastfed for the first 6 months of life, then continued breastfeeding to 2 years old or above ${ }^{[1]}$. Monitoring breastfeeding is an important part to increase the rate of breastfeeding, especially exclusively breastfeeding ${ }^{[2]}$. However, breastfeeding surveys were largely supported by maternal recall ${ }^{[3]}$. Thomas et al concerned about the data quality from those recalling method for measuring breastmilk intake for population-based studies and suggested that available analytical approaches should provide consistent results ${ }^{[4]}$.

Since the 1950s, researchers from various specialties have focused on measuring breastmilk intake via various methods, such as test weighing, Deuterium Dilution Dose-to-mother Method (DTM), observation, flowmeter, milk expression, and milk production calculation ${ }^{[5]}$. Test weighing, and DTM are more accurate than the other methods and regarded as gold standards for assessing breastmilk intake. However, test weighing methods can often interfere with the normal breastfeeding process and DTM method can be timely and costly ${ }^{[6][7]}$.

Observation method is often more convenient because they usually use medical 
63

64

indices to estimate breastmilk intake and require less operating process on mothers ${ }^{[8]}$, so the method can be easily used for assessment of infant feeding in telemedicine. But the accuracy of this method needs to be improved.

There were several studies on observation method to evaluate breastmilk intake.

Audible swallowing was considered as a valid observational indicator to determine milk intake per individual feed ${ }^{[6]}{ }^{[7][9]}$. However, there was great variation among observers, and observers should be trained to recognize feeding patterns ${ }^{[7]}$. What's more, each swallow should be recognized and counted from the beginning to the end during one breastfeeding session; meanwhile swallowing behavioral cues were not explicitly ${ }^{[7]}$. So without assistant tools, swallow counting is timely and needs great effort.

Timekeeping is easier to observe rather than counting swallows. Scott et al used breastfeeding time to estimate the amount of breastmilk consumed, and milk intake was calculated as $10 \mathrm{~g} / \mathrm{min}^{[10]}$. However, this was likely an over-simplified approach, and the definition of breastfeeding time was not strictly defined. Thomas et al reviewed that in Dewey's study estimated $24 \mathrm{~h}$ breastmilk intake equaled $100 *$ infant weight ( 3 months, $\mathrm{kg})+0.49 *$ nursing time ( $\mathrm{min}$ ), but for the calculation certain influential predictor variables should be considered, such as infant age and sex, infant feeding data, to validate and modify the volume calculations ${ }^{[4]}$. However, measuring $24 \mathrm{~h}$ breastmilk intake was not easy to perform, and breastfeeding frequency should be another contributor, and that would make measuring work complex and time-consuming.

As it is presented above, we aimed to use breastfeeding time to explore a simple observational method to estimate breastmilk intake of one breastfeeding episode and try to 
find out other influence factors to improve the accuracy of the method by establishing multilevel models in order to further apply in telemedicine in future.

\section{Materials and methods}

$$
\text { Design }
$$

This study was a cross-sectional survey conducted in a sample of mother-infant dyads.

Usually, lactation is established by 1 month., so infants aged from 1 month to 12 months were enrolled due to the stability and sustainability of breastmilk expression ${ }^{[11]} .30$ dyads were planned to be recruited in each for four groups (1-2, 3-4, 5-6, 9 -12 months) by stratified sampling.

\section{Setting}

Data of Mother-infant dyads were collected from June to July in 2015 in a clinic of Shenzhen city, in southern China.

\section{Participants}

Infants inclusion criteria were: (1) healthy 1-12-month infants born without congenital diseases; (2)infants who were fully or partially breastfeeding had no problem in sucking and swallowing; (3) routine medical examinations were carried out in the department of child health care of the maternal and child health hospital; (4) breastfeeding mothers agreed to accept and participate in the survey; (5) healthy mothers without diseases that affect lactation, including infectious diseases and any severe disease; (6) mothers who were non-smokers and nondrinkers.

\section{Measurement}

Test-weighing was used to measure milk profile per observed breastfeeding session by 
weighing infants on an accurate digital scale (Sophisticated Baby Weight Scale, Betterren Inc, Shanghai, China, resolution $2 \mathrm{~g}$ ) immediately before and after feeding with one breast. And weights before breastfeeding were adopted as infants present weights. Infants did not have their clothing removed for the weighing to minimize disruption.

\section{Data collection}

One breastfeeding session was observed in the clinic in the daytime for each participating mother. Breastfeeding was unrestrained and the mothers breastfed their infants from one breast according to their preference. Sucking behaviors of infants during breastfeeding were observed.

The following indicators in one breastfeeding session were recorded: (1) Sucking time (ST, min): represented breastfeeding time in our study, and was defined as lasting time of sucking behaviors, which was followed by swallowing, regardless of the ratio of sucking and swallowing. Breastfeeding time to comfort the infants (only latch-on) was excluded. (2) Breastmilk intake (BI, g): was equal to the infant weight gain after breastfeeding.(3) Average velocity of breastmilk intake (VB, g/min: was equal to the value of BI divided by ST. (4) Infant weight $(\mathrm{kg})$ : the infant present weight before breastfeeding.

Questionnaires were administered to determine mother-infant dyad health, maternal breast information during the breastfeeding session, and infant feeding information.

\section{Data Analysis}

All statistical analyses were performed using SPSS (Version 20.0, IBM Corporation).

Numerical variables examined for non-normal distribution, outliers, and missing data, were presented as the mean $(\overline{\mathrm{X}})$, standard deviation (SD), and minimum and maximum. 
Categorical variables were presented as numbers and proportions. T-tests were performed to investigate breastfeeding indicators in different conditions, including infant gender, infant age, infant present weight and birth weight, parity, maternal age, maternal body mass index (BMI), maternal vocation, breastfeeding side, and breastfeeding frequency per day. Distribution of BI by ST was plotted by linear regression, and Pearson Correlation test was used.

Multiple linear stepwise regressions were used to assess BI, BI per kg (infant weight), and dependent variables were effective sucking time, breastfeeding side, breastfeeding frequency per day, infant weight, infant age (day), sex, birth weight, parity, maternal age, BMI, and vocation. $\mathrm{P}<0.05$ was considered statistically significant.

\section{Results}

\section{General information}

We enrolled 181 mother-infant dyads, among which 31 were excluded, due to breastfeeding time recorded on bilateral breasts (24 mothers), birth weights less than $2500 \mathrm{~g}$ (5 infants) and ineligible breastfeeding data ( 2 infants). Considering breast side might contribute to the variances of breastmilk intake, our study collected the data of one episode, and separated the right and the left breast.

In total 150 mothers who breastfed their infants aged 1-12 months with one breast were included in the analysis. Table 1 and 2 show that average maternal age, BMI, infant birth weight and birth length was $28.47 \pm 4.19$ years, $21.54 \pm 3.85 \mathrm{~kg} / \mathrm{m}^{2}, 3291.89 \pm 415.15 \mathrm{~g}$ and $50.27 \pm 1.44 \mathrm{~cm}$ respectively. Infant age, infant weight, ST, BI and VB ranged: 30-359 day, 3.56-13.64 kg, 2-19 min, 4.00-148.00 g and 0.80-26.00 g/min respectively. Housewives accounted for $57.33 \%$ of participating mothers; primiparous women take up $67.33 \%$ of all 
participating mothers; a total of $50 \%$ of infants aged 1-4 months; infants in 1-12 months that

breastfeeding frequency per day $\geqslant 8$ accounted for $36.24 \%$.

153 Table 1 General information of measuring data of participants $(\mathrm{N}=150)$

\begin{tabular}{lccc}
\hline & $\overline{\mathrm{X}} \pm \mathrm{SD}^{\mathrm{a}}$ & Minimum & Maximum \\
\hline Maternal age $(\mathrm{year})$ & $28.47 \pm 4.19$ & 20.00 & 43.00 \\
Maternal BMI $^{\mathrm{b}}\left(\mathrm{kg} / \mathrm{m}^{2}\right)$ & $21.54 \pm 3.85$ & 15.81 & 55.47 \\
Birth weight $(\mathrm{g})$ & $3291.89 \pm 415.15$ & 2520.00 & 4900.00 \\
Birth length $(\mathrm{cm})$ & $50.27 \pm 1.44$ & 45.00 & 57.00 \\
Infant age (day) & $138.61 \pm 82.65$ & 30.00 & 369.00 \\
Infant weight $(\mathrm{kg})$ & $7.037 \pm 1.74$ & 3.56 & 13.64 \\
Sucking time (min) & $7.93 \pm 3.60$ & 2.00 & 19.00 \\
Breastmilk intake $(\mathrm{g})$ & $55.66 \pm 31.22$ & 4.00 & 148.00 \\
Average velocity of breastmilk & & & \\
intake (g/min) & $7.41 \pm 4.04$ & 0.80 & 26.00 \\
\hline
\end{tabular}

${ }^{\mathrm{a}}$ Mean \pm Standard Deviation

$155 \quad$ b Body Mass Index

Table 2 Comparisons of breastfeeding indicators according to the model's variables

\begin{tabular}{|c|c|c|c|c|}
\hline General information & $\mathrm{n}$ & $\begin{array}{l}\text { Sucking time } \\
(\mathrm{min})\end{array}$ & $\begin{array}{l}\text { Breastmilk } \\
\text { intake }(\mathrm{g})\end{array}$ & $\begin{array}{l}\text { Average velocity } \\
\text { of breastmilk } \\
\text { intake }(\mathrm{g} / \mathrm{min})\end{array}$ \\
\hline \multicolumn{5}{|l|}{ Gender } \\
\hline Male & 80 & $7.49 \pm 3.60$ & $56.08 \pm 32.52$ & $7.84 \pm 4.27$ \\
\hline Female & 70 & $8.43 \pm 3.55$ & $55.19 \pm 29.91$ & $6.93 \pm 3.73$ \\
\hline $\mathrm{p}$ & & 0.11 & 0.86 & 0.17 \\
\hline \multicolumn{5}{|l|}{ Breastfeeding side } \\
\hline Right & 77 & $8.03 \pm 3.58$ & $61.14 \pm 32.14$ & $8.12 \pm 4.48$ \\
\hline Left & 73 & $7.82 \pm 3.64$ & $49.88 \pm 29.35$ & $6.66 \pm 3.38$ \\
\hline $\mathrm{p}$ & & 0.73 & 0.03 & 0.03 \\
\hline \multicolumn{5}{|l|}{ Parity } \\
\hline Primipara & 101 & $8.19 \pm 3.71$ & $55.66 \pm 30.37$ & $7.16 \pm 3.69$ \\
\hline Multipara & 49 & $7.39 \pm 3.32$ & $55.65 \pm 33.25$ & $7.92 \pm 4.67$ \\
\hline $\mathrm{p}$ & & 0.20 & 1.00 & 0.28 \\
\hline \multicolumn{5}{|l|}{ Maternal vocation } \\
\hline Working & 64 & $7.88 \pm 3.78$ & $50.17 \pm 31.07$ & $6.60 \pm 3.65$ \\
\hline Housewife & 86 & $7.97 \pm 3.48$ & $59.74 \pm 30.88$ & $8.02 \pm 4.22$ \\
\hline $\mathrm{p}$ & & 0.88 & 0.06 & 0.03 \\
\hline \multicolumn{5}{|l|}{ Maternal age (year) } \\
\hline$\geq 30$ & 48 & $8.52 \pm 3.94$ & $56.71 \pm 32.27$ & $6.91 \pm 3.05$ \\
\hline$<30$ & 98 & $7.66 \pm 3.46$ & $56.66 \pm 30.51$ & $7.86 \pm 4.39$ \\
\hline $\mathrm{p}$ & & 0.18 & 0.99 & 0.18 \\
\hline Maternal BMI & & & & \\
\hline
\end{tabular}




\begin{tabular}{|c|c|c|c|c|}
\hline \multicolumn{5}{|l|}{$\left(\mathrm{kg} / \mathrm{m}^{2}\right)^{\mathrm{a}}$} \\
\hline$<21$ & 75 & $7.88 \pm 3.40$ & $57.53 \pm 31.61$ & $7.82 \pm 4.50$ \\
\hline$\geq 21$ & 74 & $8.12 \pm 4.13$ & $53.68 \pm 31.13$ & $7.00 \pm 3.52$ \\
\hline $\mathrm{p}$ & & 0.70 & 0.45 & 0.22 \\
\hline \multicolumn{5}{|c|}{ Infant birth weight (g) } \\
\hline$<3000$ & 25 & $8.32 \pm 3.61$ & $51.64 \pm 27.83$ & $6.37 \pm 3.22$ \\
\hline$\geq 3000$ & 125 & $7.85 \pm 3.61$ & $56.46 \pm 31.90$ & $7.62 \pm 4.16$ \\
\hline $\mathrm{p}$ & & 0.55 & 0.48 & 0.16 \\
\hline \multicolumn{5}{|c|}{ Infant age (month) } \\
\hline $1-4$ & 75 & $8.04 \pm 3.55$ & $51.20 \pm 28.68$ & $6.67 \pm 3.70$ \\
\hline $5-12$ & 75 & $7.81 \pm 3.67$ & $60.12 \pm 33.17$ & $8.15 \pm 4.25$ \\
\hline $\mathrm{p}$ & & 0.70 & 0.08 & 0.02 \\
\hline \multicolumn{5}{|c|}{$\begin{array}{l}\text { Breastfeeding } \\
\text { frequency/day }\end{array}$} \\
\hline$\geq 8$ & 54 & $8.07 \pm 3.51$ & $53.00 \pm 27.93$ & $6.88 \pm 3.60$ \\
\hline$<8$ & 95 & $7.78 \pm 3.63$ & $56.81 \pm 32.98$ & $7.73 \pm 4.27$ \\
\hline $\mathrm{p}$ & & 0.63 & 0.48 & $0.22^{-}$ \\
\hline
\end{tabular}

${ }^{\mathrm{a}}$ Body Mass Index

Distribution of breastfeeding indicators and Simple variable analysis

Figure 1 shows the scatter plot of BI distribution by ST, and BI was positively correlated with ST $(r=0.59, \mathrm{p}<0.05)$. ST accounted for only $35 \%\left(\mathrm{R}^{2}=0.35\right)$ of the variation in milk intake.

Table 2 indicates that, average BI from the right breast was higher than the left $(61.14 \pm 32.14$ vs. $49.88 \pm 29.35 \mathrm{~g}, \mathrm{p}<0.05)$; average VB from the right breast was greater than the left $(8.12 \pm 4.48$ vs. $6.66 \pm 3.38 \mathrm{~g} / \mathrm{min}, \mathrm{p}<0.05)$; average $\mathrm{VB}$ in housewives was higher than working mothers $(8.02 \pm 4.22$ vs. $6.60 \pm 3.65 \mathrm{~g} / \mathrm{min}, \mathrm{p}<0.05)$; and VB was higher in infants of

5-12 months compared to those of $1-4$ months $(8.15 \pm 4.25$ vs. $6.67 \pm 3.70 \mathrm{~g} / \mathrm{min}, \mathrm{p}<0.05)$.

\section{Multilevel models}

As shown in Table 3, all four multilevel models had statistical significance: in Model 1 to estimate $\mathrm{BI}$ in infants of $1-12$ months $\left(\mathrm{r}=0.62, \mathrm{R}^{2=0.39}\right), \mathrm{ST}(\mathrm{b}=5.15 \pm 0.57)$, breastfeeding side $(b=-9.48+4.11)$ and infant weight $(b=3.58 \pm 1.19)$ remained significant; in Model 2 to 
171

estimate BI in infants of $1-4$ months $\left(r=0.69, \mathrm{R}^{2}=0.48\right)$, ST $(\mathrm{b}=4.45 \pm 0.69)$, breastfeeding side $(b=-20.47 \pm 5.11)$ and birth weight $(b=15.47 \pm 6.53)$ were included; in Model 3 to estimate BI per kg (infant weight) in infants of $1-12$ months $\left(r=0.70, \mathrm{R}^{2}=0.49\right), 5$ variables remained significant: ST ( $b=0.78 \pm 0.08)$, breastfeeding side $(b=-1.34 \pm 0.59)$, infant weight $(b=-0.64 \pm 0.17)$, maternal vocation $(b=1.51 \pm 0.60)$, and maternal BMI $(b=-1.54 \pm 0.60)$; in Model 4 to estimate BI per kg (infant weight) in infants of $1-4$ months $\left(r=0.73, \mathrm{R}^{2}=0.53\right)$, ST $(\mathrm{b}=0.84 \pm 0.12)$, breastfeeding side $(\mathrm{b}=-2.85 \pm 0.88)$ and maternal BMI $(\mathrm{b}=-2.40 \pm 0.89)$ remained significant .

Table 3 Multilevel models to estimate breastmilk intake /velocity of breastmilk intake $(\mathrm{b} \pm \mathrm{SD})^{\mathrm{a}}$

\begin{tabular}{|c|c|c|c|c|}
\hline & \multicolumn{2}{|c|}{ Breastmilk intake } & \multicolumn{2}{|c|}{ Breastmilk intake per $\mathrm{kg}$} \\
\hline & $1-12 \mathrm{mo}$ & $1-4 \mathrm{mo}$ & $1-12 \mathrm{mo}$ & $1-4 \mathrm{mo}$ \\
\hline & Model 1 & Model 2 & Model 3 & Model 4 \\
\hline Constant & $4.52 \pm 12.64$ & $20.75 \pm 14.59$ & $8.72 \pm 2.08$ & $10.84 \pm 2.20$ \\
\hline Sucking time & $5.15 \pm 0.57$ & $4.45 \pm 0.69$ & $0.78 \pm 0.08$ & $0.84 \pm 0.12$ \\
\hline Breastfeeding side & $-9.48 \pm 4.11$ & $-20.47 \pm 5.11$ & $-1.34 \pm 0.59$ & $-2.85 \pm 0.88$ \\
\hline Infant weight & $3.58 \pm 1.19$ & & $-0.64 \pm 0.17$ & \\
\hline Birth weight & & $15.47 \pm 6.53$ & & \\
\hline Maternal vocation & & & $1.51 \pm 0.60$ & \\
\hline Maternal BMI & & & $-1.54 \pm 0.60$ & $-2.40 \pm 0.89$ \\
\hline$r / R^{2}$ & $0.62 / 0.39$ & $0.69 / 0.48$ & $0.70 / 0.49$ & $0.73 / 0.53$ \\
\hline $\mathrm{p}$ (model) & $<0.05$ & $<0.05$ & $<0.05$ & $<0.05$ \\
\hline
\end{tabular}

${ }^{\text {a }}$ Stepwise regressions were used to establish all models. Variance Inflation Factors (VIF) were used for Collinearity Diagnostics, and all the included variables' VIFs were $<2$.

${ }^{\mathrm{b}}$ Body Mass Index

\section{Discussion}

Sucking time and breastmilk intake 
Here we confirmed that there was a significantly positive relationship between sucking time and breastmilk intake $(\mathrm{r}=0.57, \mathrm{p}<0.05)$, and our multilevel model in infants of $1-12$ months showed that sucking time played a critical role in estimating breastfeeding intake $(b=5.15, r=0.62, p<0.05)$. Thus, it's possible that sucking time per feed is adopted to establish equations to estimate breastmilk intake. There are few studies on the methods for evaluating breastmilk intake of one breastfeeding by breastfeeding time. Only in the studies of swallow evaluation one breastfeeding session was observed ${ }^{[6]}$.

In our study breastfeeding time was recorded based on sucking and swallowing during breastmilk ejection, and the sucking time ranged from 2 to 19 minutes. So it should be pointed out that if the method in this study is applied to assess breastmilk intake, breastfeeding time within certain range is recommended. Also, Gardner et al found that obvious ejections occurred in the first 8 minutes in a 12-minute expression session ${ }^{[13]}$; in Scott's research, the amount of breastmilk intake was calculated as $10 \mathrm{~g} /$ minute to a maximum of $100 \mathrm{~g}^{[10]}$. Besides, our models are used to estimate one breastfeeding session on unilateral breast, so if infants are breastfed on both breasts, time needs to be counted for each breast to get a complete picture of estimated total BI.

\section{Factors from infants, mothers and feeding practice}

Analyzing breastmilk intake per minute in one feed appeared more meaningful than the quantity of breastmilk consumed according to the single factor analysis, and this inferred that the factors from mother-child dyads affected breastmilk intake, namely infants' ability of breastmilk intake and maternal ability of milk expression. Breastfeeding is a coordinated process of maternal milk expression and infant's behaviors of sucking and swallowing, and the factors that influence maternal lactation status and infant's ability of sucking and swallowing will affect milk intake ${ }^{[14]}$. Therefore, when models were established to estimate breastmilk intake of unilateral breast, factors influencing the short-term ability of breastmilk production and intake should be considered. Here we divided factors into three groups: factors from infants, maternal factors and feeding practice, which may have short-term effect. Factors from infants:

According to Thomas et al, infant age should be a key determinant of breastfeeding practice $^{[4]}$. However, infant weight but not age had significant relationship with 
breastfeeding intake in Model 1 for 1-12-month infants. Therefore, our results suggested that infant weight may provide a better fit model than infant age. Also, Dewey et al found that infant weight could also explained the variance of breastmilk intake of 24 hours besides total breastfeeding time ${ }^{[12]}$. Dualde et al built the equation $(140 \mathrm{~mL} / \mathrm{kg}$ (infant weight) per day) employed infant weight referring human milk intake equations recommended by USEPA (United States Environmental Protection Agency) ${ }^{[16]}$.

Thomas et al reported that infant gender should be taken into account in assessing 24-hour volume of breastfeeding ${ }^{[4]}$. Our data indicated that gender did not play a significant role in the multilevel models to estimate breastmilk intake of one feed.

Maternal factors:

In the present study, whether working played significantly in one of our multilevel models. Gilany et al reported that housewife was one of the independent predictors of exclusive breastfeeding ${ }^{[15]}$, which supported that housewives tended to express more breastmilk. Maternal postpartum BMI was negatively associated with breastmilk intake. Diana et al got the similar finding that maternal BMI was not a protective factor of milk production for breastfed infants ${ }^{[17]}$.

Feeding factors:

Our multilevel models showed breast side was a key contributor of breastmilk intake and the right side produced more breastmilk. This may be due to the fact that mostly the right breast was the dominant breast in the breastfeeding mothers participating in this study. Cox et al reported that $24 \mathrm{~h}$ milk production of right breast was more than left breast in participating mothers in most time, and it suggested that the right breast was the breast side of dominance ${ }^{[19]}$.Also, using bilateral breasts separately to assess milk production was reported by Kent et $\mathrm{al}^{[20]}$. There was a hypothesis that right breasts produced more milk in right-handed mothers due to more blood flow of the right side ${ }^{[11]}$. Therefore, it's necessary to distinguish breast sides in estimating BI of one episode, and information of maternal handedness which was not available in the study should be also recorded.

Thomas suggested approaches to classify feeding style, including exclusively breastfeeding and combination feeding ${ }^{[4]}$, but our study used breastfeeding frequency for replacement and found that feeding frequency was not a significant factor in the multilevel 
models. Although breastfeeding frequency can be an indicator of assessing breastfeeding, further research still needs to separate exclusively breastfeeding and combination feeding ${ }^{[18]}$. Better fit models

We found birth weight impacted breastmilk intake in younger infants while present weight impacted breastmilk intake in older infants, and Whitehead et al had the same result of factors influencing breastmilk output ${ }^{[21]}$. Meanwhile, there were improvements in the correlation coefficients of Models for younger infants (1-4 months), compared to the Models for all infants (1-12 months). Therefore, factors affecting lactation changed with age groups, and it's necessary to analyze and establish models according to different age groups.

The correlation coefficients of models to estimate breastmilk intake per kg (infant weight) had improved, and Model $4\left(\mathrm{r}=0.73, \mathrm{R}^{2}=0.53\right)$ to estimate breastmilk intake per $\mathrm{kg}$ for 1-4-month infants was the optimal equation. Thus some solutions, such as dependent variable conversion, could be taken to get better statistical result.

\section{Strengths and Limitations}

Establishing multilevel models based on sucking time to estimate breastmilk intake has greatly improved the convenience of measuring milk intake, and the effectiveness of models for specific age group of infants have improved compared to using sucking time alone. This makes it possible to use breastfeeding time to evaluate breastmilk intake in telemedicine.

However, further research is needed to improve the accuracy of the measurement: (1) the key factor: feeding style (exclusively breastfeeding, mixed feeding, and complementary feeding) influencing short-term milk synthesis should be addressed; (2) adequate sample should be collected in each month of infants; (3) milk ejection patterns (discrete few, discrete many, non-discrete and pulsatile) can affect volume of milk expression in different breastfeeding sessions with identical breastfeeding time ${ }^{[22]}$, so estimation models should distinguish and adopt frequency of ejections.

\section{Conclusions}

There was a significant relationship between sucking time and breastmilk intake per breastfeeding session $(r=0.57, \mathrm{p}<0.05)$. The best fit model in our study was to estimate breastmilk intake per $\mathrm{kg}$ (infant weight) for $1-4$-month infants $\left(\mathrm{r}^{2}=0.53\right)$. Sucking time as the 
key factor and other factors including infant birth weight/ birth weight, breast side, maternal BMI and vocation significantly had effect on breastmilk intake and the factors that played significant varied according to different age groups. However, the accuracy of the estimation needs to be improved for further application in telemedicine.

\section{List of abbreviations}

BI (breastmilk intake)

ST (sucking time)

BMI (body mess index)

DTM (Deuterium Dilution Dose-to-mother Method)

VB (average velocity of breastmilk intake)

\section{Declarations}

Ethics approval and consent to participate

The Ethics Committee of Capital Institute of Pediatrics (SHERLLM2020013) approved the study according to the International Organizations of Medical Sciences on "Human biomedical research international guidelines" and ethical principles from "the Declaration of Helsinki". We obtained written informed consent from the mothers on the behalf of the infant participants involved in this study.

\section{Consent for publication}

Not applicable

Availability of data and materials

The datasets used and/or analyzed during the current study are available from the corresponding author on reasonable request.

\section{Competing interests}

The authors declare that they have no competing interests

Funding

This research was funded by the Mengniu Arla Nutrition Research Center. Authors' contributions

Ni Jia, Yaohua Dai, Jianhua Gong and Lu Zhao designed research; Jianhua Gong and Lu 
Zhao conducted research. Ni Jia analyzed data and wrote the paper. Mengxue Xu and Meixian Zhang edited the manuscript. Ni Jia and Yaohua Dai had primary responsibility for final content. All authors read and approved the final manuscript. Acknowledgements

We thank Luohu Maternal and Child Health Hospital for their dedicated field work in enrolling participants, questionnaire survey, and performing weighing infants. We also thank Changping, Qinghai and Kunming Maternal and Child Health Hospital for supporting the research.

Thanks to Huanmei Zhang, Yanfeng Zhang, Hao Peng and Medjaden for editing our manuscript.

\section{References}

1. WHO. Global Strategy for Infant and Young Child Feeding. 2003.

2. Mulol H, Coutsoudis A. Limitations of maternal recall for measuring exclusive breastfeeding rates in South African mothers. Int Breastfeed J. 2018;13:19. doi: $10.1186 / \mathrm{s} 13006-018-0159-8$.

3. Bailey RL, Catellier DJ, Jun S, Dwyer JT, Jacquier EF, Anater AS, Eldridge AL. Total Usual Nutrient Intakes of US Children (Under 48 Months): Findings from the Feeding Infants and Toddlers Study (FITS) 2016. J Nutr. 2018;148(9S):1557S-1566S. doi: 10.1093/jn/nxy042.

4. Thomas BL, Gross R, Messito MJ, Deierlein A, Katzow M, Woolf K. Concerns about Current Breast Milk Intake Measurement for Population-Based Studies. J Acad Nutr Diet 2018;118(10):1827-31. doi: 10.1016/j.jand.2018.06.010.

5. Hartmann PE, Saint L. Measurement of Milk Yield in Women. Journal of Pediatric Gastroenterology and Nutrition. 1984;3(2):270-4. doi: 10.1097/00005176-19840300000019

6. Chantal L, Susan J H. A Noninvasive Method for Determining Patterns of Milk Intake in the Breast-Fed Infant. Journal of Pediatric Gastroenterology and Nutrition. 1989;9: 481-487. doi: 10.1097/00005176-198911000-00015.

7. Scanlon KS, Alexander MP, Serdula MK, Davis MK, Bowman BA. Assessment of infant 
feeding: the validity of measuring milk intake. Nutr Rev. 2002;60(8):235-51. doi: $10.1301 / 002966402320289368$.

8. Jia N, Dai Y. The research progress on measurement methods of infant breastmilk intake. Chinese Journal of Woman and Child Health Research. 2020;31(1):1-5. doi: 10.1089/bfm.2011.0096.

9. Côté-Arsenault D, McCoy TP. Reliability and validity of swallows as a measure of breast milk intake in the first days of life. J Hum Lact. 2012;28(4):483-9. doi: $10.1177 / 0890334412451364$.

10. Scott J, Davey K, Ahwong E, Devenish G, Ha D, Do L. A Comparison by Milk Feeding Method of the Nutrient Intake of a Cohort of Australian Toddlers. Nutrients. 2016;8(8): E501. doi: 10.3390/nu8080501.

11. Xielian G, Yu S, Meihua Zh. Breastfeeding and Human Lactation (6th edition). Beijing: People's Medical Publishing House. 2021.

12. Dewey KG, Heinig MJ, Nommsen LA, Lonnerdal B. Maternal versus infant factors related to breast milk intake and residual milk volume: the DARLING study. Pediatrics. 1991;87(6):829-37.

13. Gardner H, Kent JC, Prime DK, Lai CT, Hartmann PE, Geddes DT. Milk ejection patterns remain consistent during the first and second lactations. Am J Hum Biol. 2017;29(3). doi: 10.1002/ajhb.22960.

14. Chen L, Lucas RF, Feng B. A Novel System to Measure Infants' Nutritive Sucking During Breastfeeding: the Breastfeeding Diagnostic Device (BDD). IEEE J Transl Eng Health Med. 2018;6:2700208. doi: 10.1109/JTEHM.2018.2838139.

15. El-Gilany AH, Shady E, Helal R. Exclusive breastfeeding in Al-Hassa, Saudi Arabia. Breastfeed Med. 2011;6(4):209-13. doi: 10.1089/bfm.2010.0085.

16. Dualde $\mathrm{P}$, Pardo $\mathrm{O}$, Corpas-Burgos F, Kuligowski J, Gormaz M, Vento M, Pastor A, Yusà V. Biomonitoring of bisphenols A, F, S in human milk and probabilistic risk assessment for breastfed infants. Sci Total Environ. 2019;668:797-805. doi: 10.1016/j.scitotenv. 2019.03.024.

17. Diana A, Haszard JJ, Houghton LA, Gibson RS. Breastmilk intake among exclusively breastfed Indonesian infants is negatively associated with maternal fat mass. Eur J Clin 
Nutr. 2019;73(8):1206-1208. doi: 10.1038/s41430-019-0458-1.

18. Sayres S, Visentin L. Breastfeeding: uncovering barriers and offering solutions. Curr Opin Pediatr. 2018.30(4):591-596. doi: 10.1097/MOP.0000000000000647.

19. Cox DB, Owens RA, Hartmann PE. Blood and milk prolactin and the rate of milk synthesis in women. Exp Physiol. 1996;81(6):1007-20. doi: 10.1113/expphysiol.1996.sp 003985.

20. Kent JC, Gardner H, Lai CT, Hartmann PE, Murray K, Rea A, Geddes DT. Hourly Breast Expression to Estimate the Rate of Synthesis of Milk and Fat. Nutrients. 2018;10(9):1144. doi: $10.3390 /$ nu10091144.

21. Whitehead RG, Rowland MG, Hutton M, Prentice AM, Müller E, Paul A. Factors influencing lactation performance in rural Gambian mothers. Lancet. 1978;2(8082):178-81. doi: 10.1016/s0140-6736(78)91920-7.

22. Prime DK, Geddes DT, Hepworth AR, Trengove NJ, Hartmann PE. Comparison of the patterns of milk ejection during repeated breast expression sessions in women. Breastfeed Med. 2011;6(4):183-90. doi: 10.1089/bfm.2011.0014. 
Figures

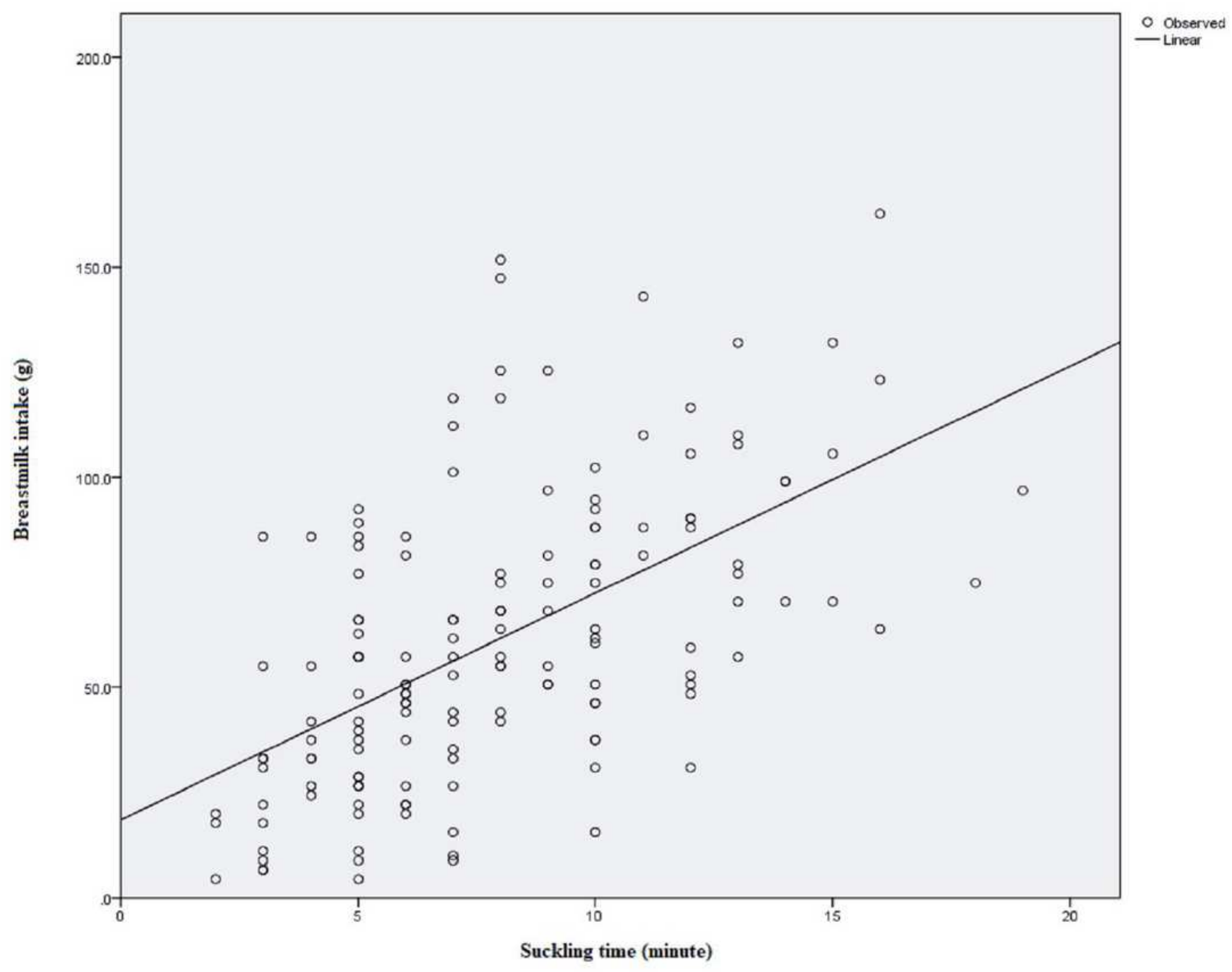

Figure 1

Distribution of BI by ST in one breastfeeding session. Figure Legends: Scatter plot of BI distribution by suckling time, and breastmilk intake was positively related to $S T(r=0.57, p<0.05)$. Linear regression was used, and there was a linear relationship between suckling time and breastmilk intake $(b=5.37, p<0.05)$. 